Published in final edited form as:

Cancer Epidemiol Biomarkers Prev. 2007 June ; 16(6): 1065-1069.

\title{
Association of COMT Val108/158Met Genotype with Smoking Cessation in a Nicotine Replacement Therapy Randomized Trial
}

\author{
Elaine C. Johnstone ${ }^{1}$, Katherine M. Elliot ${ }^{1}$, Sean P. David ${ }^{3}$, Michael F.G. Murphy ${ }^{2}$, Robert T. \\ Walton $^{1,4}$, and Marcus R. Munafó ${ }^{5}$ \\ ${ }^{1}$ Department of Clinical Pharmacology, University of Oxford, Oxford, United Kingdom ${ }^{2}$ Childhood Cancer \\ Research Group, University of Oxford, Oxford, United Kingdom ${ }^{3}$ The Warren Alpert Medical School of Brown \\ Medical School and Memorial Hospital of Rhode Island, Rhode Island ${ }^{4}$ Medical Research Council \\ Laboratories, Banjul, Gambia ${ }^{5}$ Department of Experimental Psychology, University of Bristol, Bristol, United \\ Kingdom
}

\begin{abstract}
We investigated the association of catechol O-methyltransferase (COMT) genotype with abstinence following a smoking cessation attempt among a large cohort of smokers who attempted to quit using either the nicotine transdermal patch or placebo and were followed up over an 8-year period following their initial cessation attempt. In addition, we examined the possible moderating influence of sex on any association. The genotype $\times$ treatment interaction effect at 12 -week follow-up indicated a greater benefit of active nicotine replacement treatment compared with placebo on likelihood of abstinence in the COMT Met/Met genotype group (33\% versus $12 \%$ ), in comparison to the Met/Val + Val/Val group (22\% versus $16 \%$ ). Our results indicate that COMT genotype may moderate the effect of active transdermal nicotine patch compared with placebo, with reduced relative benefit of nicotine replacement therapy in individuals with Met/Val or Val/Val genotype. Our data follow an emerging pattern of results suggesting that genetic variation in the dopamine pathway may provide a future basis for tailored smoking cessation therapies, but indicate that different genes influencing various components of this pathway may have different effects on response to smoking cessation pharmacotherapy.
\end{abstract}

\section{Introduction}

Over 1 billion people worldwide continue to smoke cigarettes (1), and it is estimated that 500 million people alive today will be killed by smoking (2). Although many smokers report a desire to quit, only a small number embark on a cessation attempt in any given year (3). The majority of those that do attempt to quit eventually relapse to smoking, most within 12 months or less of the cessation attempt (3). Smoking behavior is known to be under a degree of genetic influence (4). Pharmacogenetic research has a number of aims, including to determine whether the efficacy of different pharmacotherapies for the treatment of nicotine dependence is influenced by inherited variation in drug-metabolizing enzymes and drug targets $(5,6)$. To date, two pharmacogenetic trials of nicotine replacement therapy (NRT) have been conducted (713).

Based on the neurobiology of reward (14), several pharmacogenetic analyses have focused on genes in the dopamine pathway $(7,8,11,15,16)$. Nicotine is the main addictive component of tobacco smoking and stimulates the release of dopamine in the nucleus accumbens, which is

Requests for reprints: Marcus R. Munafò, Department of Experimental Psychology, University of Bristol, 12a Priory Road, Bristol BS8 1TU, United Kingdom, Phone: 44-117-9546841; Fax: 44-117-9288588. E-mail: marcus.munafo@bristol.ac.uk 
partly responsible for its reinforcing effects $(17,18)$. Polymorphisms in genes in the dopamine pathway are therefore strong candidates for influencing smoking behavior phenotypes, including smoking cessation and treatment response. The enzyme catechol $O$ methyltransferase (COMT) is of relevance in pharmacogenetic studies of nicotine dependence and treatment outcome due to its presence in dopaminergic brain regions and its role as a key enzyme in the degradation and inactivation of extraneuronally released dopamine $(19,20)$. The $\mathrm{Val}^{108 / 158}$ Met polymorphism is located on exon 3 of the COMT gene (rs4680) and is a G1947A transition that results in the substitution of a valine $(\mathrm{Val}, \mathrm{G})$ by a methionine (Met, $\mathrm{A}$; ref. 21) at codon 108/158, for S-COMT and MB-COMT, respectively (22). The Met (A) allele confers low activity of the gene, resulting in a 3- to 4-fold reduction in COMT enzyme activity (23), which is hypothesized to result in relatively greater dopamine activity as a result of reduced inactivation with lower enzyme activity (23).

Two recent investigations have shown a positive association with COMT genotype and smoking behavior, but only in women. Colilla et al. (16) found that women who were homozygous for the low-activity Met allele were significantly more likely to be abstinent from smoking at the end of a period of NRT. Another recent study by Beuten et al. (24) found that a COMT haplotype, including the $\mathrm{Val}^{108 / 158}$ Met polymorphism, had a significant association with nicotine dependence, conferring protection in females but not males. However, a number of studies have failed to show any association between COMT genotype and tobacco consumption (25), and smoking initiation, persistence, and cessation (26). An evaluation of nonreplicated results involving COMT and nicotine dependence was carried out by Redden et al. (27). The authors critically reviewed two contradicting studies, analyzing often-cited reasons for nonreplication, such as type 1 error, low statistical power, and population stratification. Although they found no definitive answer, they concluded that the failure to replicate the results from the first study was likely to be due either to low statistical power to detect a small effect, or the effect of heterogeneity.

The evidence that the association of COMT genotype with smoking behavior may differ in males and females $(16,24)$ is consistent with evidence for individual differences in COMT activity in males and females, which is mediated by differences in estrogen metabolism. COMT is involved in the breakdown of catechol estrogens to estrone (28). Estrogen is also a regulator of COMT promoter activity (29), and COMT activity is lower in the brain (19) and blood (30) of women, due to inactivation by estrogen. Therefore, any effect of COMT genotype on smoking cessation may vary between women and men due to this sex-specific difference in COMT activity. There is also growing wider interest in sex differences in smoking cessation and treatment response, although the evidence is somewhat conflicting (31).

To the best of our knowledge, no studies to date have investigated COMT genotype and smoking cessation among treatment-seeking smokers using NRT. We therefore investigated the association of COMT genotype with abstinence following a smoking cessation attempt among a large cohort of smokers who attempted to quit using either the NRT transdermal patch or placebo and were followed up over an 8-year period following their initial cessation attempt. In addition, we examined the possible moderating influence of sex on any association.

\section{Materials and Methods}

\section{Participants}

Participants in the original study included $N=1,686$ patients from general practice (GP) surgeries in Oxfordshire, United Kingdom, who participated in a double-blind, randomized, placebo-controlled trial of the nicotine transdermal patch between June 1991 and March 1992 (the Patch study; refs. 32,33). The inclusion criteria for this study were that participants smoked at least 15 cigarettes a day and were aged between 25 and 65 years. 
In 1999 to 2000, participants were recontacted and invited to enter the study. Of the $N=1,686$ participants enrolled in the Patch study, $n=154$ subjects were unavailable because they could not be located (moved, emigrated, or untraceable) or were deceased. Invitation letters were sent to the remaining $n=1,532$ participants, and those interested in joining the study were given an appointment with a nurse at each participant's GP surgery, during which a short questionnaire was given and a 10-mL blood sample was collected. Blood samples were successfully collected on $n=755$ (49\%) participants. The methods for recruitment, allocation, and randomization of the Patch $(32,33)$, and the 8-year follow-up (Patch II) studies $(7,11,32$, 33) have been comprehensively described.

\section{Treatment}

In the Patch study, participants were randomly assigned to wear active nicotine or placebo patches for 12 weeks by prior random allocation of study numbers to each intervention group and sequential allocation of a study number to participants on entry. Participants were assessed by a study nurse at $1,4,8,12,26$, and 52 weeks. Active and placebo patches were identical as prepared by the manufacturer, and all investigators and patients were blinded to treatment allocation. The main outcome measure was self-reported abstinence, which was confirmed with salivary cotinine and exhaled carbon monoxide $(\mathrm{CO})$ measurement.

Ethical approval was obtained from the Anglia and Oxford Multicentre Research Ethics Committee and from the 86 Local Research Ethics Committees covering the areas of residence of the participants in the Patch study.

\section{Abstinence Verification}

Abstinence at 1, 4, and 8 weeks was confirmed by an expired $\mathrm{CO}$ reading $\leq 10 \mathrm{ppm}$ and at 12 , 26 , and 52 weeks by a salivary cotinine level $\leq 20 \mathrm{ng} / \mathrm{mL}$ ( $89 \%$ of cases) or expired CO reading $\leq 10 \mathrm{ppm}$. Saliva cotinine was assayed by gas chromatography in the Department of Preventive Medicine at St. Bartholomew's Medical College (London, United Kingdom).

\section{Genotyping}

Participants were genotyped for the COMT Val ${ }^{108 / 158}$ Met G1947A polymorphism using methods described in detail elsewhere (25) and briefly here. PCR was carried out using an allele-specific, two-tube primer method. The reaction mixture contained $0.7 \mu \mathrm{mol} / \mathrm{L}$ of each COMT primer, sequences ATGGTGGATTTCGCTGGCG and ATGGTGGATTTCGCTGGCA for the $\mathrm{G}$ and A sequence-specific primers, respectively, and GATGTCCTGGGACGCTCC for the common reverse, $0.3 \mu \mathrm{mol} / \mathrm{L}$ of each control primer, $\sim 25 \mathrm{ng}$ of DNA, $300 \mu \mathrm{mol} / \mathrm{L}$ deoxynucleotide triphosphates, $1.5 \mathrm{mmol} / \mathrm{L} \mathrm{MgCl}_{2}$, and 0.1 units of Taq polymerase in a final volume of $10 \mu \mathrm{L}$. After an initial denaturation step for $1 \mathrm{~min}$ at $96^{\circ} \mathrm{C}$, thermocycling consisted of 5 cycles of $96^{\circ} \mathrm{C}$ for $20 \mathrm{~s}, 70^{\circ} \mathrm{C}$ for $45 \mathrm{~s}, 72^{\circ} \mathrm{C}$ for $25 \mathrm{~s}$, followed by 21 cycles of $96^{\circ} \mathrm{C}$ for $25 \mathrm{~s}, 65^{\circ} \mathrm{C}$ for $50 \mathrm{~s}, 72^{\circ} \mathrm{C}$ for $30 \mathrm{~s}$, then 4 cycles of $96^{\circ} \mathrm{C}$ for $30 \mathrm{~s}, 55^{\circ} \mathrm{C}$ for $60 \mathrm{~s}$, and $72^{\circ} \mathrm{C}$ for $90 \mathrm{~s}$. A final step of $20^{\circ} \mathrm{C}$ for $5 \mathrm{~min}$ was followed by cooling of samples to $4^{\circ} \mathrm{C}$. PCR products were separated on a $1 \%$ agarose gel at $200 \mathrm{~V}$ for $24 \mathrm{~min}$ and visualized using ethidium bromide. In order for samples to be valid, a 220-bp control product had to be formed in both allele-specific reactions. A homozygote was indicated by the presence of a 350-bp product in one of the reactions, and a heterozygote was represented by a 350-bp product in both of the reactions.

\section{Statistical Analysis}

Biochemically verified point-prevalence 7-day abstinence, at 12-week (end of treatment; EOT) and 26-week follow-up, were the primary outcome measures. Participants lost to follow-up 
were assumed to have relapsed to smoking and coded as such in outcome analyses (i.e., intent to treat analyses).

Separate models of outcome at 12-week and 26-week follow-up were generated within a logistic regression framework because pharmacotherapy was available only during the treatment phase. The full models included age, sex, socioeconomic status, and nicotine dependence at baseline in the first step, treatment group (active patch, placebo patch) and COMT genotype (Met/Met, Met/Val + Val/Val) in the second step, and interaction terms for treatment $\times$ sex, treatment $\times$ genotype, sex $\times$ genotype, and treatment $\times$ sex $\times$ genotype in the third step. Terms were entered using the backward conditional method, with term removal conditional on $P>0.10$, with the exception of main effects of treatment and genotype which were entered using the enter method. COMT Met/Val and Val/Val genotypes were combined to increase statistical power and given evidence for recessive effects on functional activity (23).

All analyses were done using the Statistical Package for the Social Sciences (v. 12.0). An $\alpha$ level of 0.05 was maintained throughout the analysis.

\section{Statistical Power}

Of the $n=724$ participants in the final study population, $n=363$ received active patch, and $n=361$ received placebo patch. The sample size was adequate to detect a risk ratio of 1.6 at 12 -week follow-up and 1.8 at 26-week follow-up, with a power of 0.80 , for a main effect of genotype on cessation.

\section{Results}

\section{Characteristics of Participants}

Of the $n=749$ participants who were successfully genotyped for the COMT Val ${ }^{108 / 158} \mathrm{Met}$ G1947A polymorphism, we retained $n=741$ of European ancestry to avoid potential population stratification. There were missing data on $n=17$ participants, resulting in a final sample for analysis of $n=724$ smokers ( $59 \%$ female) of European ancestry. The mean age of participants was 43 years (SD, 10; range, 25-65). Participants were older than non-participants $(M=43$ years versus $M=42$ years; $P=0.002)$, more likely to be female $(59 \%$ versus $53 \% ; P=0.010)$ and more likely to have quit for a year in the trial $(14 \%$ versus $6 \% ; P<0.001)$. There was no difference in the frequency of abstinence from smoking between the genotype groups at either follow-up (12-week, $P=0.25 ; 26$-week, $P=0.87)$. COMT genotype frequencies did not deviate significantly from Hardy-Weinberg Equilibrium $(P=0.46)$.

\section{Analysis of Smoking Cessation Outcomes}

The main effect of treatment was significant at 12 -week $(P<0.001)$ and 26 -week $(P=0.004)$ follow-up, indicating an increased likelihood of abstinence on active NRT relative to placebo, as previously reported $(32,33)$. There was evidence for a genotype $\times$ treatment interaction effect at 12 -week follow-up $(P=0.050)$, but this term was not significant at 26-week follow-up and not retained in the model, and a $Z$ test indicated that the interaction effect at 26-week followup differed significantly from that at 12 -week follow-up $(Z=21.87, P<0.001)$. Age was positively associated with the likelihood of abstinence at 12-week $(P=0.013)$ and 26 -week $(P=0.010)$ follow-up. Higher SES was marginally associated with an increased likelihood of abstinence at 12 -week follow-up $(P=0.090)$, but was not retained in the model at 26-week follow-up. Finally, nicotine dependence was not retained in the model at 12-week follow-up but was included at 26-week follow-up, indicating a trend for lower dependence to be associated with an increased likelihood of abstinence at 26 -week follow-up $(P=0.085)$. The main effect of genotype was not significant at either 12 -week $(P=0.353)$ or 26 -week $(P=$ 
0.914) follow-up. No main effects or interaction terms involving sex were retained in either model.

The significant genotype $\times$ treatment interaction effect at 12 -week follow-up indicated a greater benefit of active NRT treatment compared with placebo on likelihood of abstinence in the COMT Met/Met genotype group (33\% versus $12 \%$ ), in comparison to the Met/Val + Val/Val group (22\% versus $16 \%$ ). These data are presented graphically in Fig. 1.

Baseline characteristics by treatment group are presented in Table 1 . The final logistic regression models for abstinence at both 12-week and 26-week follow-up are presented in Table 2.

\section{Discussion}

Our results indicate that $C O M T$ genotype may moderate the effect of active transdermal nicotine patch compared with placebo. We observed a greater benefit of active NRT treatment compared with placebo on the likelihood of abstinence in the COMT Met/Met genotype group, in comparison to those in either the Met/Val or Val/Val groups. These effects were only observed at 12-week follow-up (EOT), which suggests that this represents a short-term pharmacogenetic effect. This effect attenuated and was no longer significant at 26-week follow-up and did not seem to differ between males and females.

A recent study represents the only previous pharmacogenetic study of COMT genotype and smoking cessation. Berrettini et al. (15) found that a COMT haplotype of two single nucleotide polymorphisms (including $\mathrm{Val}^{108 / 158} \mathrm{Met}$ ) predicted favorable outcome of bupropion pharmacotherapy for smoking cessation, although no differences in this association were observed between men and women. However, the small numbers in some of the haplotype groups in this study suggest that these results require confirmation in a larger study. To the best of our knowledge, ours is the first study to evaluate the association of COMT genotype and smoking cessation among treatment-seeking smokers using NRT.

It is possible, albeit speculative, that the effects of COMT genotype on NRT treatment response that we observed are mediated by individual differences in withdrawal symptoms following cessation, and the extent to which withdrawal symptoms are attenuated by NRT may account for the relatively greater efficacy of patch in individuals with Met/Met genotype compared with those with Met/Val or Val/Val genotypes. There is evidence that some smokers seem to self-medicate to reduce cognitive dysfunction (e.g., inability to concentrate) during nicotine abstinence (34). There is strong evidence that the prefrontal cortex modulates certain components of working memory and attention (35), which is, in part, mediated by dopaminergic neurotransmission (36), and consequently modulated by COMT genotype (37). Unfortunately, data do not exist to allow us to test this possibility directly in our sample.

The lack of a moderating effect of sex on our observed COMT $\times$ treatment interaction is interesting given observations of decreased COMT activity in brain and blood of women (19, 30 ). One case-control study has reported a sex-specific association with nicotine dependence in females (24), but these investigators did not report associations with smoking cessation outcomes, whereas a further study in females only suggests an association with abstinence among individuals who completed a course of NRT (16). However, the dopamine degradative pathway is, as stated above, complex and is under the influence of the dopamine transporter, MAO, aldehyde dehydrogenase, and potentially other gene products. Future studies should investigate variation in multiple candidate genes in this pathway. It is also possible that dopaminergic candidate genes may exert differential effects on response to smoking cessation pharmacotherapy as a function of the different brain regions that may underpin the mechanisms mediating these associations (e.g., ventral striatum versus prefrontal cortex). Future large-scale 
studies will be required to afford sufficient power to simultaneously investigate the role of multiple genetic variants in treatment response and the effects of potential moderating variables on these associations, such as sex. Neuroimaging studies may also shed light on the mechanisms that subserve these associations and may further serve to test the likely efficacy of potential novel pharmacotherapies.

Our results indicate that $C O M T$ genotype may moderate the effect of active transdermal nicotine patch compared with placebo, with reduced benefit of standard NRT in individuals with either Met/Val or Val/Val genotypes. Further studies are needed to examine possible biobehavioral mechanisms that may mediate our observed genotype $\times$ treatment interaction, to provide biological plausibility for these data. In addition, replication will be required before the association identified in our data can be considered to be robust. Future studies should also seek to examine potential interactions between genotype and sex with adequate sample size to afford sufficient power to detect higher level interactions. However, these results do follow an emerging pattern of results, suggesting that genetic variation in the dopamine pathway may provide a future basis for tailored smoking cessation therapies $(5,6)$.

\section{Acknowledgements}

Grant support: Cancer Research United Kingdom Program Grant.

\section{References}

1. Proctor RN. Tobacco and the global lung cancer epidemic. Nat Rev Cancer 2001;1:82-6. [PubMed: 11900255]

2. WHO. WHO framework convention on tobacco control. Geneva: WHO Press; 2003.

3. Hughes JR. Motivating and helping smokers to stop smoking. J Gen Intern Med 2003;18:1053-7. [PubMed: 14687265]

4. Munafo MR, Clark T, Johnstone EC, Murphy M, Walton RT. The genetic basis for smoking behavior: a systematic review and meta-analysis. Nicotine Tob Res 2004;6:583-97. [PubMed: 15370155]

5. Munafo MR, Lerman C, Niaura R, Shields AE, Swan GE. Smoking cessation treatment: pharmacogenetic assessment. Curr Opin Mol Ther 2005;7:202-8. [PubMed: 15977416]

6. Munafo MR, Shields AE, Berrettini WH, Patterson F, Lerman C. Pharmacogenetics and nicotine addiction treatment. Pharmacogenomics 2005;6:211-23. [PubMed: 16013953]

7. Johnstone EC, Yudkin PL, Hey K, et al. Genetic variation in dopaminergic pathways and short-term effectiveness of the nicotine patch. Pharmacogenetics 2004;14:83-90. [PubMed: 15077009]

8. Lerman C, Jepson C, Wileyto EP, et al. Role of functional genetic variation in the dopamine D2 receptor (DRD2) in response to bupropion and nicotine replacement therapy for tobacco dependence: results of two randomized clinical trials. Neuropsychopharmacology 2006;31:231-42. [PubMed: 16123753]

9. Lerman C, Wileyto EP, Patterson F, et al. The functional mu opioid receptor (OPRM1) Asn ${ }^{40} \mathrm{Asp}$ variant predicts short-term response to nicotine replacement therapy in a clinical trial. Pharmacogenomics J 2004;4:184-92. [PubMed: 15007373]

10. Munafo MR, Johnstone EC, Wileyto EP, Shields PG, Elliot KM, Lerman C. Lack of association of 5-HTTLPR genotype with smoking cessation in a nicotine replacement therapy randomized trial. Cancer Epidemiol Bio-markers Prev 2006;15:398-400.

11. Yudkin P, Munafo MR, Hey K, et al. Effectiveness of nicotine patches in relation to genotype in women versus men: randomised controlled trial. Br Med J 2004;328:989-90. [PubMed: 15033882]

12. Dahl JP, Jepson C, Levenson R, et al. Interaction between variation in the D2 dopamine receptor (DRD2) and the neuronal calcium sensor-1 (FREQ) genes in predicting response to nicotine replacement therapy for tobacco dependence. Pharmacogenomics J 2006;6:194-9. [PubMed: 16402081]

13. Malaiyandi V, Lerman C, Benowitz NL, Jepson C, Patterson F, Tyndale RF. Impact of CYP2A6 genotype on pretreatment smoking behavior and nicotine levels from and usage of nicotine replacement therapy. Mol Psychiatry 2006;11:400-9. [PubMed: 16402128] 
14. Robinson TE, Berridge KC. The neural basis of drug craving: an incentive-sensitization theory of addiction. Brain Res Brain Res Rev 1993;18:247-91. [PubMed: 8401595]

15. Berrettini WH, Wileyto EP, Epstein L, et al. Catechol- $O$-methyltransferase (COMT) gene variants predict response to bupropion therapy for tobacco dependence. Biol Psychiatry 2007;61:111-8. [PubMed: 16876132]

16. Colilla S, Lerman C, Shields PG, et al. Association of catechol- $O$-methyltransferase with smoking cessation in two independent studies of women. Pharmacogenet Genomics 2005;15:393-8. [PubMed: 15900212]

17. Balfour DJ. The neurobiology of tobacco dependence: a preclinical perspective on the role of the dopamine projections to the nucleus accumbens. Nicotine Tob Res 2004;6:899-912. [PubMed: 15801566]

18. Pontieri FE, Tanda G, Orzi F, Di Chiara G. Effects of nicotine on the nucleus accumbens and similarity to those of addictive drugs. Nature 1996;382:255-7. [PubMed: 8717040]

19. Chen J, Lipska BK, Halim N, et al. Functional analysis of genetic variation in catechol- $O$ methyltransferase (COMT): effects on mRNA, protein, and enzyme activity in postmortem human brain. Am J Hum Genet 2004;75:807-21. [PubMed: 15457404]

20. Akil M, Kolachana BS, Rothmond DA, Hyde TM, Weinberger DR, Kleinman JE. Catechol-Omethyltransferase genotype and dopamine regulation in the human brain. J Neurosci 2003;23:200813. [PubMed: 12657658]

21. Jonsson EG, Nothen MM, Grunhage F, et al. Polymorphisms in the dopamine D2 receptor gene and their relationships to striatal dopamine receptor density of healthy volunteers. Mol Psychiatry 1999;4:290-6. [PubMed: 10395223]

22. Lachman HM, Papolos DF, Saito T, Yu YM, Szumlanski CL, Weinshilboum RM. Human catechol$O$-methyltransferase pharmacogenetics: description of a functional polymorphism and its potential application to neuropsychiatric disorders. Pharmacogenetics 1996;6:243-50. [PubMed: 8807664]

23. Shield AJ, Thomae BA, Eckloff BW, Wieben ED, Weinshilboum RM. Human catechol $O$ methyltransferase genetic variation: gene resequencing and functional characterization of variant allozymes. Mol Psychiatry 2004;9:151-60. [PubMed: 14966473]

24. Beuten J, Payne TJ, Ma JZ, Li MD. Significant association of catechol- $O$-methyltransferase (COMT) haplotypes with nicotine dependence in male and female smokers of two ethnic populations. Neuropsychopharmacology 2006;31:675-84. [PubMed: 16395295]

25. McKinney EF, Walton RT, Yudkin P, et al. Association between polymorphisms in dopamine metabolic enzymes and tobacco consumption in smokers. Pharmacogenetics 2000;10:483-91. [PubMed: 10975602]

26. David SP, Johnstone E, Griffiths SE, et al. No association between functional catechol $O$ methyltransferase $1947 \mathrm{~A}>\mathrm{G}$ polymorphism and smoking initiation, persistent smoking or smoking cessation. Pharmacogenetics 2002;12:265-8. [PubMed: 11927842]

27. Redden DT, Shields PG, Epstein L, et al. Catechol- $O$-methyltransferase functional polymorphism and nicotine dependence: an evaluation of nonreplicated results. Cancer Epidemiol Biomarkers Prev 2005;14:1384-9. [PubMed: 15941945]

28. Ball P, Knuppen R, Haupt M, Breuer H. Interactions between estrogens and catechol amines. 3. Studies on the methylation of catechol estrogens, catechol amines and other catechols by the catechol$O$-methyltransferases of human liver. J Clin Endocrinol Metab 1972;34:736-46. [PubMed: 5012775]

29. Xie T, Ho SL, Ramsden D. Characterization and implications of estrogenic down-regulation of human catechol- $O$-methyltransferase gene transcription. Mol Pharmacol 1999;56:31-8. [PubMed: 10385681]

30. Weinshilboum RM, Otterness DM, Szumlanski CL. Methylation pharmacogenetics: catechol $O$ methyltransferase, thiopurine methyltransferase, and histamine $N$-methyltransferase. Annu Rev Pharmacol Toxicol 1999;39:19-52. [PubMed: 10331075]

31. Munafo MR, Bradburn M, Bowes L, David SP. Are there sex differences in transdermal nicotine replacement therapy patch efficacy? A meta-analysis Nicotine Tob Res 2004;6:769-76.

32. Imperial Cancer Research Fund. Effectiveness of a nicotine patch in helping people stop smoking: results of a randomised trial in general practice. Br Med J 1993;306:1304-8. [PubMed: 8518571] 
33. Imperial Cancer Research Fund. Randomised trial of nicotine patches in general practice: results at one year. Br Med J 1994;308:1476-7. [PubMed: 8019282]

34. Lerman C, Audrain J, Tercyak K, et al. Attention-deficit hyperactivity disorder (ADHD) symptoms and smoking patterns among participants in a smoking-cessation program. Nicotine Tob Res 2001;3:353-9. [PubMed: 11694203]

35. Volkow ND, Fowler JS, Wang GJ, Goldstein RZ. Role of dopamine, the frontal cortex and memory circuits in drug addiction: insight from imaging studies. Neurobiol Learn Mem 2002;78:610-24. [PubMed: 12559839]

36. Seamans JK, Yang CR. The principal features and mechanisms of dopamine modulation in the prefrontal cortex. Prog Neurobiol 2004;74:1-58. [PubMed: 15381316]

37. Flint J, Munafo MR. The endophenotype concept in psychiatric genetics. Psychol Med 2007;37:16380. [PubMed: 16978446]

38. Russell MA, Peto J, Patel UA. The classification of smoking by factorial structure of motives. J R Stat Soc 1974;137:313-46. 

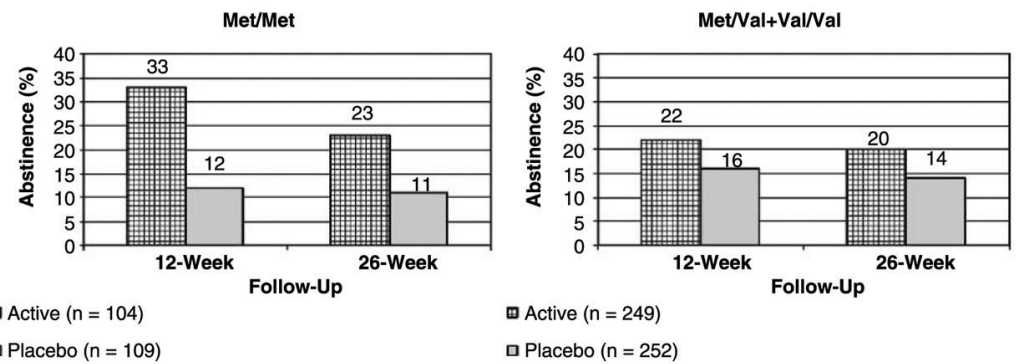

口Placebo $(n=109)$

$\square$ Placebo $(n=252)$

Figure 1.

Abstinence at 12-wk and 26-wk follow-up, by COMT genotype and treatment group. The proportion of participants abstinent in the active and placebo treatment groups is presented, grouped by COMT genotype. Participants in the Met/Val + Val/Val group seem to derive less benefit from active NRT compared with placebo during the treatment phase (12-wk followup) than those in the Met/Met group. This difference has attenuated and is no longer statistically significant at 26-wk follow-up. 
Table 1

Demographic characteristics and COMT genotype frequency by treatment condition

\begin{tabular}{lcc}
\hline & Active $(\boldsymbol{n}=\mathbf{3 6 3})$ & Placebo $(\boldsymbol{n}=\mathbf{3 6 1})$ \\
\hline Age (y), $M \pm$ SD & $42 \pm 10$ & $43 \pm 10$ \\
Sex (male), $n(\%)$ & $152(42)$ & $144(40)$ \\
Socioeconomic status, $n(\%)$ & $11(3)$ & $8(2)$ \\
$\quad$ I-professional & $112(31)$ & $717(33)$ \\
II-managerial & $86(24)$ & $77(21)$ \\
IIIa-nonmanual & $75(21)$ & $58(16)$ \\
IIIb-manual & $63(17)$ & $26(7)$ \\
IV-semiskilled & $16(4)$ & $15 \pm 4$ \\
V-unskilled & $15 \pm 5$ & $109(30)$ \\
Nicotine dependence, $M \pm$ SD & & $166(46)$ \\
COMT genotype, $n(\%)$ & $114(31)$ & $86(24)$ \\
Met/Met & $165(46)$ & \\
Met/Val & $84(23)$ & \\
Val/Val & & \\
\hline
\end{tabular}

NOTE: Baseline measures do not differ significantly between the active and placebo groups $(P>0.28)$. 
Table 2

Logistic regression models of abstinence at 12-wk and 26-wk follow-up

\begin{tabular}{|c|c|c|c|c|}
\hline & \multicolumn{2}{|c|}{ 12-wk follow-up } & \multicolumn{2}{|c|}{ 26-wk follow-up } \\
\hline & OR $(95 \% \mathrm{CI})$ & $P$ & OR $(95 \%$ CI $)$ & $P$ \\
\hline Age & $1.02(1.00-1.04)$ & 0.013 & $1.03(1.01-1.05)$ & 0.010 \\
\hline Socioeconomic status & $0.88(0.76-1.02)$ & 0.090 & & \\
\hline Nicotine dependence ${ }^{*}$ & & & $0.96(0.92-1.00)$ & 0.085 \\
\hline Treatment & $3.53(1.75-7.13)$ & $<0.001$ & $1.81(1.21-2.72)$ & 0.004 \\
\hline$C O M T^{\dagger}$ genotype & $1.37(0.70-2.69)$ & 0.353 & $0.98(0.64-1.50)$ & 0.914 \\
\hline$C O M T^{\dagger} \times$ treatment & $0.43(0.19-1.00)$ & 0.050 & & \\
\hline
\end{tabular}

NOTE: Covariates controlled for in the model included age, sex, socioeconomic status, and nicotine dependence. Only those terms retained in the models are presented. Interaction terms for $C O M T \times \operatorname{sex}$, treatment $\times$ sex, and $C O M T \times \operatorname{sex} \times$ treatment were not retained in the model for any follow-up assessments.

Abbreviations: OR, odds ratio; 95\% CI, 95\% confidence interval.

Nicotine dependence measured using the Horn-Russell Scale (38)

${ }^{\dagger}$ Reference group is Met/Met. 